\title{
Research on the Influencing Factors and Cultivation Strategies of New Professional Farmers' Training Willingness from the Perspective of Public Policy Science
}

\author{
Yong-qi ZHANG \\ The second Campus of North China Electric Power University \\ Baoding, Hebei, China
}

\begin{abstract}
Keywords: Public Policy Science; New Professional Farmers; Willingness to Train; Influencing Factors; Conclusions and Recommendations.
\end{abstract}

\begin{abstract}
The new-type professional farmers are the backbone of promoting modern agriculture. Intensifying the training of new professional farmers' skills is an urgent requirement in solving the "three rural issues". In this paper, a survey was conducted on 483 farmers in Baoding. The situation of peasant education and training in Baoding City was thoroughly understood, and factors influencing the willingness of new-type professional peasants were investigated. It was concluded that gender, educational level, and views on the role of science and technology in agriculture will become factors influencing the willingness of farmers to participate in the training of new professional farmers. Based on this, it puts forward the countermeasures and suggestions for the cultivation of new professional farmers in Baoding City.
\end{abstract}

\section{Introduction}

Farming heavyweights, outline of the country. The issue of agriculture, rural areas and farmers has always been the focus and key issue of rural work. In 2007, the Central No. 1 document formally proposed the training of new farmers. The Central Document No. 1 in 2013 established the core of cultivating new professional farmers as a means of promoting modern agricultural construction. In 2014, the Central Document No. 1 also emphasized the need to increase education and training for new professional farmers. In 2016, the Central No. 1 document proposed that: basically form a professional peasant education and training system, cultivate professional farmers into the dominant force in the construction of modern agriculture. Vigorously carrying out vocational skills training for farmers is in line with the call of the Central Government. General Secretary Xi Jinping emphasized the eradication of poverty, improvement of people's livelihood, and the gradual realization of common prosperity in the Politburo meeting. This is an essential requirement of socialism. Vigorously carrying out vocational skills training for farmers is the foundation for China to realize modern agriculture and is an important measure for poverty alleviation.

\section{Research Methods}

The paper adopts the questionnaires, with the new farmers in the surrounding areas of Baoding City as the research object, to discuss the influencing factors and cultivation strategies of new professional farmers' training willingness.

According to the purpose of the survey, the interview content was set up, the questionnaire was carefully prepared, the form of the farmer's home was adopted, the interview was carried out, and the questionnaire was sent to the respondents to fill in the answers. Finally, we collected and analyzed the questionnaire.

This paper studies the influencing factors of the willingness of new professional farmers to train. There are two kinds of results for the willingness of new-type professional farmers to train: the first result is "unwilling" and the second is "willing". If the dependent variable has only two options, you can select the Logit Model or the Probit Model. Because the two options of "unwilling" and "willing" are discrete, the Logit Model or the Probit Model is chosen. 
The purpose of this paper is to explore the individual characteristics of the new-type professional farmer, to understand and master the new type of agriculture, training experience and expected training conditions, the impact of the factors on their willingness to participate in the training of new professional farmers, and to verify the robustness of the impact. In this study, whether or not it is willing to participate in the training of a new type of professional farmer training is a dependent variable. Because the willingness of new-type professional farmer training is a classified variable whose values are "willingness" and "unwillingness", so the model is firstly applied to the orderly use of Logit. Firstly, the independent variables were gradually increased and the regression analysis was carried out by controlling the range of independent variables; secondly, we used the Ordered Probit Model and the OLS Model to perform regression analysis on the three independent variables, and analyzed the results to the former Ordered Logit Model. The regression results obtained were compared, and the robustness of the regression results was tested. Finally, a more reliable regression analysis result was obtained.

\section{Results and Discussion}

After issuing questionnaires, collating the questionnaires, and analyzing the data with Stata, the following results were obtained:

In the Ordered Logit model 1 of the individual attribute property variables of the new professional farmers, the "education level" variable has a significant positive effect on the willingness of new-type professional farmers to train, indicating that among the new professional farmers, if they have a high level of education, they are more willing to participate in the training of new-type professional farmers; people with low degrees are unwilling to participate in new professional farmer training. This is consistent with the assumption that the highly educated people may have years of learning. They can feel the role of knowledge in agricultural production and life, and therefore have higher enthusiasm. On the contrary, farmers who are less educated may be affected by traditional farming ideas and have no intention of accepting new types of agricultural technology. They are less enthusiastic about participating in training. We noticed that the model has a limited degree of interpretation, which means that in the sample of this paper, the individual attribute characteristics of the new-type professional farmer have very limited ability to interpret the results of training willingness [1].

In the Ordered Logit model 2 with new variables added, after adding the knowledge of the new type of agriculture and grasping the situation variables on the basis of Model 1, the influence of the "education level" variable on the explanatory variables is still significantly positive. However, the impact on the use of pesticides, feeds, fertilizers, etc. is not significant. At the same time, the four variables "on the role of science and technology in agriculture", "awareness of the grain market", "degree of attention to agricultural information", and "acquisition of use of agricultural machinery" are explained. The positive effects of the model are fully consistent with the assumptions of the second, third, fourth, and sixth. The reason may be that the new professional farmers have better grasp of the new type of agriculture and are more willing to learn knowledge to improve the efficiency of agricultural production and are more willing to participate in the training of new professional farmers. At this time, the R2 of the model is increased to 0.644 , which means that the understanding and mastery of the new-type professional farmer on the new type of agriculture can further explain the changes in the willingness of the new professional farmers to train about $48 \%$ $(0.644-0.161=0.483)$. This shows that in the sample interval of this paper, the knowledge and mastery of the new-type agriculture variables have a high explanatory capacity for the new professional farmers' willingness to train.

In the Ordered Logit model 3 with new variables added, after adding training experience and desired training status variables on the basis of model 2, the two variables of "education level" and "mastery of agricultural machinery use" have no significant effect on the explanatory variables. It shows that this variable is not an explanatory variable of stable effect. The variables of "Views on the Role of Science and Technology in Agriculture" and "Understanding of Food Market Quotes" are still positive for the new professional farmers' willingness to train. It further confirms the hypothesis 2 
and hypothesis 3. At the same time, the significant influence of the two variables "Is the training meets the requirements" and "Expected training costs" were added to the explanatory variables, and the "Impression of whether the training meets the requirements" effect was positive, which confirms the hypothesis 7.

Among them, the three variables of "the view of the role of science and technology in agriculture", "the understanding of the market situation of food" and "think whether the training can meet the requirements" have a significant positive impact on the training willingness of new professional farmers, and these effects are stable. We suspect that this is mainly because new-type professional farmers can understand that science and technology are the primary productive forces, they want to learn some agricultural technology in training, so as to improve the efficiency of agricultural production, and want to know more about the grain market and strengthen their own agricultural products. Market circulation, which increases income, is therefore more willing to participate in training; in addition, the "expected training costs" has a significant negative impact on the willingness of new professional farmers to train, which is inconsistent with the results we have assumed, and therefore requires more in-depth Investigate and demonstrate to draw the right conclusions.

At this point, we see that R2 has increased to 0.781 , which means that the training experience and the expected training situation variables can further explain the changes in the community comprehensive evaluation results of about $13 \%(0.781-0.644=0.137)$. This indicates that within the sample interval of this paper, community attribute variables have a high explanatory power for community comprehensive evaluation.

In addition, we should also see that the "expected training cost" in Model 3 is negative for the coefficient of influence of the explanatory variables, and it is more significant. This is completely inconsistent with hypothesis 8 . Combined with the interview results, the reason may be that agriculture is a basic industry, and the state attaches great importance to agricultural development. New-type professional farmers hope to receive free or low-cost training funded by the government, so they will have an impact on the willingness of new professional farmers to train, and there may be other reasons that cause the negative impact of the variable. However, this sample data cannot be reflected.

In order to verify the stability of the model 3, the Oprobit Model and the OLS Model were used for the regression analysis of all the explanatory variables. The regression results show that several significant variables in Model 3 have a high degree of stability.

\section{Conclusion}

Through the basic description analysis, interaction analysis and regression analysis of the data obtained from this survey, we can draw the following conclusions:

First, the characteristics of the new-type professional farmers have a direct impact on the training willingness, but they can affect the perceptions of the new-type professional farmers on the role of technology in agricultural production and the conditions for participation in training, and thus indirectly affect the willingness to train. Therefore, it is necessary to be more cautious in the selection and training of new-type professional farmers. The emphasis should be placed on those aged between 30 and 50 years of age, with high educational attainment or secondary technical schools and above, and with certain agricultural production experience, and encourage them to participate in new professional farmers training.

Secondly, the understanding of new-type occupational farmers about the new type of agriculture, such as "views on the role of science and technology in agriculture" and "awareness of the food market situation" and other factors will significantly affect the willingness of new-type professional farmers to train. The information obtained in the interview also supports this analysis result. From the survey results, most of the new-type occupational farmers think that science and technology have a great role in agriculture. They all hope to learn new agriculture in training. Learning some of the latest agricultural information and food market conditions, so to encourage emerging professional farmers to participate in training, it is recommended to promote new agricultural technology, teach the latest agricultural information and food market. 
Third, training experience and expected training conditions will have a certain impact on training willingness. If the new professional farmers have participated in the training, believe that the training can meet the requirements, and feel that the training brings them the convenience of agricultural production, then they will be willing to go to the training again. From the perspective of expected training costs, new professional farmers are more likely to participate in the government funded free or low-cost training; from the expected training content, the new professional farmers hope to learn a lot of farming techniques and appropriate amount in training. From the perspective of expected training methods, the most desirable training methods for new professional farmers are on-site teaching, followed by classroom teaching, and emerging professional farmers do not want training methods to be network distance education. Therefore, it is possible to carry out free or low-cost training on planting and breeding techniques in the form of on-site teaching, which will attract many new professional farmers to participate.

\section{Suggestions for Training}

Based on the existing conclusions, we propose the following suggestions:

\subsection{The government:}

1. Increase the propaganda of new professional farmers' training policies. Farmers who are willing to participate in training are related to whether or not they participate in training. Therefore, the government's relevant leading agencies at all levels must increase publicity on policies for new-type professional farmer training so that more people can understand the new policy.

2. Increase financial support and build a development platform. In terms of financial support, the first is to promote rural financial innovation to extend and lean toward new professional farmers, and formulate credit loan methods suitable for new professional farmers. The second is the establishment of government-specific guidance funds for new-type professional farmer training, which are used to pay various expenses required for the development of new professional farmer training and the compensation for lost work due to participation in training. In terms of platform construction, the first is to build a venture financing platform, and actively guide financial institutions to increase their support for the investment of credit funds for new professional farmers' entrepreneurial projects; the second is to build a service coordination platform. We will further establish and improve the organizational structure for the service of new-type professional farmers, effectively solve the problems of new-professional farmer entrepreneurs' medical protection and land circulation, and ensure their entrepreneurship.

\subsection{Training institutions:}

1. Adjust training content and pay attention to regional scale agricultural science and technology implantation. Before training, training institutions should go deep into the homes of farmers who are willing to participate in the training, understand the actual conditions of planting and management in detail, combine the contents of urgent requirements, adjust the training content in time, and arrange the corresponding agriculture in accordance with the principle of light and rapid relief. Scientific and technological training content, and the corresponding agricultural science and technology form a centralized breakthrough and the effect of the scale of popularization [2].

2. Promote integration of training and employment. Starting from the three training links of training, docking and tracking, we will do a good job in skills training, job development and employment promotion. The first is to further increase professional skills training. It is necessary to pay close attention to the pre-employment skills training of the shortage of technical workers, as well as the skills upgrading training of the in-service personnel of the enterprises, and to better grasp the self-employment training of new professional farmers and realize the integration of training and employment. The second is to further improve the job docking. Actively promote cross-regional cooperation, regularly carry out the docking of information on sources of new-type professional farmers' training bases and information on shortages of jobs in agriculture, gradually establish a long-term service mechanism for employment of enterprises, and raise the degree of organization of 
agricultural services. The third is to further improve the follow-up services. For the migrant workers entered as a whole, through the implementation of the recruitment enterprise scheduling regular meeting, tour inspection guidance and visits and sympathy to the three systems, the receiving unit handles recruitment procedures, wages, labor time, signed labor contracts, etc. Regulate the employment behavior and promptly urge [3].

\section{References}

[1] Hao Tianjun. Research on the training needs of new professional farmers [D]. Henan Agricultural University, 2017. (In Chinese)

[2] Wu Yixiong. Influencing Factors and Countermeasures of New-type Occupational Farmers' Agricultural Production Willingness Based on Binary Logistic Model [J]. Contemporary Economic Management, 2016, 38 (11): 40-49. (In Chinese)

[3] Zhu Qiwei, Mi Songhua, Huang Lili, Huang Hexiao. Study on the willingness and influencing factors of new professional farmers participating in skills training-An empirical analysis based on large-scale farming farmers [J]. Journal of Zhejiang Agricultural Sciences, 2014, 26 (05): 1361-1367. (In Chinese) 